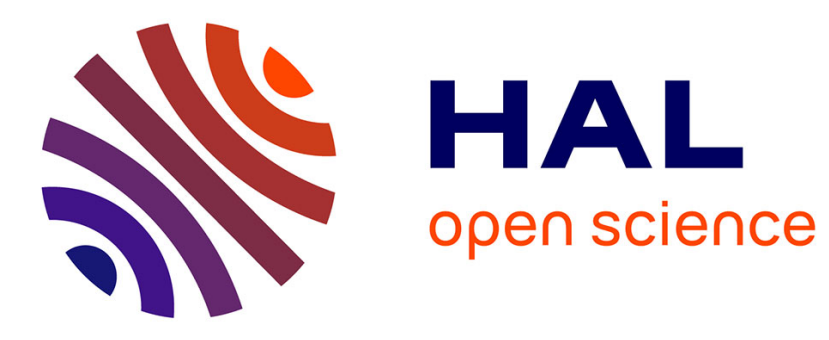

\title{
Low-speed optic-flow sensor onboard an unmanned helicopter flying outside over fields
}

Guillaume Sabiron, Paul Chavent, Thibaut Raharijaona, Patrick Fabiani, Franck Ruffier

\section{- To cite this version:}

Guillaume Sabiron, Paul Chavent, Thibaut Raharijaona, Patrick Fabiani, Franck Ruffier. Low-speed optic-flow sensor onboard an unmanned helicopter flying outside over fields. IEEE ICRA - IEEE International Conference on Robotics and Automation, May 2013, Karlsruhe, Germany. hal-00820264

\section{HAL Id: hal-00820264 \\ https://hal.inria.fr/hal-00820264}

Submitted on 3 May 2013

HAL is a multi-disciplinary open access archive for the deposit and dissemination of scientific research documents, whether they are published or not. The documents may come from teaching and research institutions in France or abroad, or from public or private research centers.
L'archive ouverte pluridisciplinaire HAL, est destinée au dépôt et à la diffusion de documents scientifiques de niveau recherche, publiés ou non, émanant des établissements d'enseignement et de recherche français ou étrangers, des laboratoires publics ou privés. 


\title{
Low-speed optic-flow sensor onboard an unmanned helicopter flying outside over fields*
}

\author{
Guillaume Sabiron $^{1,2}$, Paul Chavent ${ }^{2}$, Thibaut Raharijaona ${ }^{1}$, Patrick Fabiani ${ }^{2}$ and Franck Ruffier ${ }^{1}$
}

\begin{abstract}
The 6-pixel low-speed Visual Motion Sensor (VMS) inspired by insects' visual systems presented here performs local 1-D angular speed measurements ranging from $1.5^{\circ} / \mathrm{s}$ to $25^{\circ} / \mathrm{s}$ and weighs only $2.8 \mathrm{~g}$. The entire optic flow processing system, including the spatial and temporal filtering stages, has been updated with respect to the original design. This new lightweight sensor was tested under free-flying outdoor conditions over various fields onboard a $80 \mathrm{~kg}$ unmanned helicopter called ReSSAC. The visual disturbances encountered included helicopter vibrations, uncontrolled illuminance, trees, roads, and houses. The optic flow measurements obtained were finely analyzed online and also offline, using the sensors of various kinds mounted onboard ReSSAC. The results show that the optic flow measured despite the complex disturbances encountered closely matched the approximate ground-truth optic flow.
\end{abstract}

\section{INTRODUCTION}

Finding means of sensing the optic flow onboard unmanned aerial and terrestrial vehicles has been a key research topic during the last few decades. The term "optic flow" is used to denote the angular velocity (in $\%$ ) of the images sweeping backward across the visual field. Several flight control systems based on optic flow cues have been constructed so far for performing hazardous tasks such as hovering and landing on a moving platform [1], avoiding obstacles [2][4], following terrain [5] and tracking a moving target [6]. Insects are able to navigate safely in complex, unfamiliar environments thanks to the built-in abilities they have developed and improved during several hundred millions of years. Based on the findings obtained at our Laboratory on the fly's visual system [7], several versions of the 2-pixel Local Motion Sensor (LMS) [8]-[12] were developed, using an algorithm introduced by [13], [14], which was later called the "time of travel scheme" (see [15], [16]). Several visionbased systems have been previously designed to measure the optic flow onboard UAVs (Unmanned Aerial Vehicles) [17]-[19] and in particular in the $1.5-25^{\circ} / \mathrm{s}$ range [3], [6], [20]. Most of these visual systems were quite demanding in terms of their computational requirements and/or their

\footnotetext{
* This research was supported by the French Aerospace Lab (ONERA, Systems Control and Flight Dynamics department), CNRS Institutes (Life Science; Information Science; Engineering Science and Technology), AixMarseille University, Astrium Satellites and ESA under NPI contract.

1 G. Sabiron, T. Raharijaona and F. Ruffier are with Aix-Marseille University, CNRS, Institute of Movement Science, Biorobotics Dept., UMR7287, 13288, Marseille, France \{Thibaut.Raharijaona, Franck.Ruffier\}@univ-amu.fr.

${ }^{2}$ G. Sabiron, P. Chavent and P. Fabiani are with the French Aerospace Lab (ONERA, Systems Control and Flight Dynamics -DCSD-), 31055 Toulouse, France \{Guillaume.Sabiron, Paul.Chavent, Patrick.Fabiani\}@onera.fr
}

weight or were not very well characterized, except for the optical mouse sensors [21], with which a standard error of approximately $\pm 5^{\circ} / \mathrm{s}$ around $25^{\circ} / \mathrm{s}$ was obtained in a $\pm 280^{\circ} / \mathrm{s}$ overall range. However, very few studies have been published so far to our knowledge in which visual motion sensors have been implemented and tested outdoors onboard an unmanned aircraft subject to vibrations, where the illuminance cannot be easily controlled (see [2] in the case of linear 1-D motion sensors and see [3], [5], [21], [22] in that of 2-D optic flow sensors). It therefore seemed to be worth testing the reliability of the present 1-D optic flow-based visual sensor onboard a free-flying helicopter in terms of its resolution, accuracy, sensitivity and invariance to contrast in real outdoor environments. The output signals produced by this tiny 6-pixel visual motion sensor dedicated to gauging low visual angular speeds was tested onboard the ONERA's unmanned helicopter called ReSSAC (ReSSAC stands in French for Recherche et Sauvetage par Système Autonome Coopérant) travelling over an uninhabited village, where the dynamics and vibrations involved were assessed using real data acquired (see Fig. 1). In Section 2, the basic

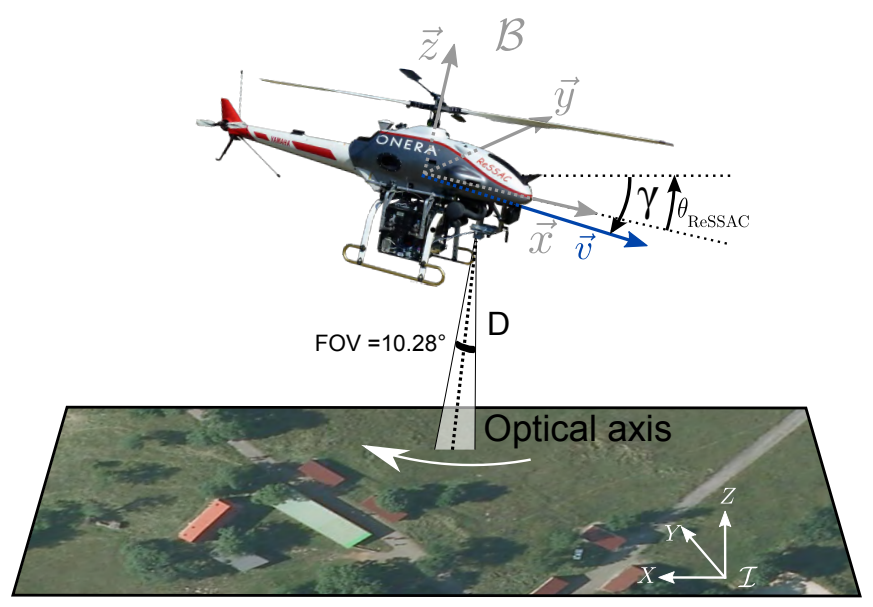

Fig. 1. 1-D optic flow generated by ReSSAC flying at the velocity $\vec{v}$ with the pitch angle $\theta_{\operatorname{ReSSAC}} . D$ is the actual distance from the sensor to the ground and $\gamma$, the flight path angle, is defined as the angle between the local horizontal plane and the velocity vector's orientation. Aerial view of the flight environment obtained on geoportail.fr

equations of the optic flow are defined. Section 3 gives a brief description of the 6-pixel 1-D visual motion device and outlines the processing algorithm as well as the optical and electrical assembly involved. The results of the outdoor experiments performed in basic forward flight condition are presented and analyzed in Section 4. 


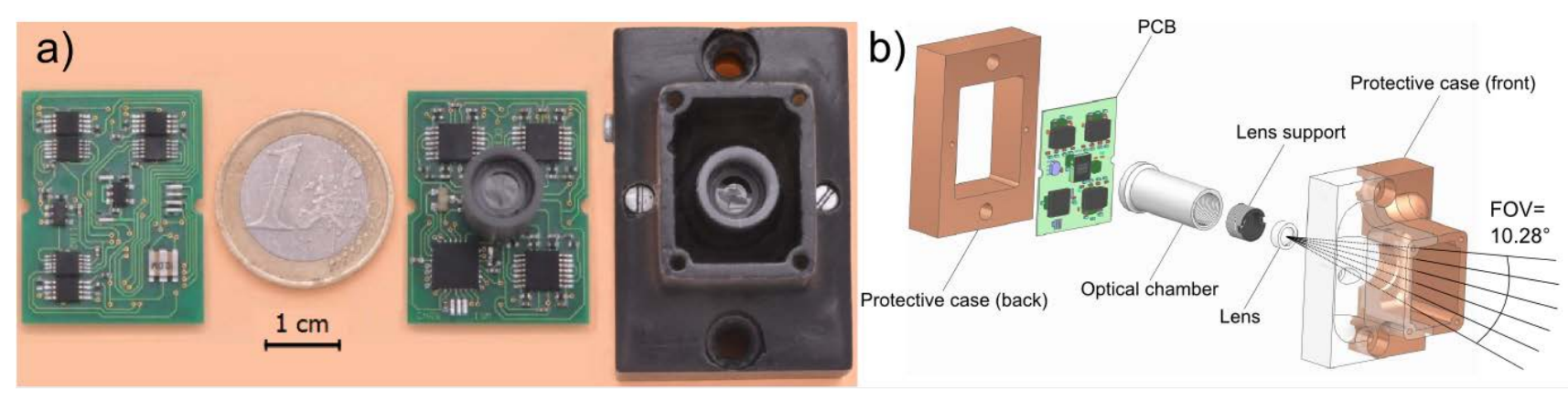

Fig. 2. (a) Top and bottom view of the electronic board (size: $33 \times 40 \mathrm{~mm}$ ) of a low-speed visual motion sensor with its lens mounted on the LSC photosensor array. The custom-made protective case is presented on the right. (b) Exploded view of the complete assembly, including the custom-made protective case (front and back), the electronic board, and the optical assembly (lens, lens support, optical chamber).

\section{Definition of The GRound-TRuth Optic Flow}

The ground-truth optic flow $\omega_{\text {grd-trh }}$ can be described as the sum of the two distinct components defined by [23], i.e. the translational and rotational optic flow:

$$
\omega_{\text {grd-trh }}=\omega_{T}+\omega_{R}
$$

The translational optic flow depends on the linear velocity $v$ expressed in the inertial frame $\mathcal{I}$ associated with the vector basis $(\vec{X}, \vec{Y}, \vec{Z})$, the distance from the ground $D$ and the elevation angle $\Phi$ (i.e., the angle between the gaze direction and the heading direction).

$$
\omega_{T}=\frac{v}{D} \cdot \sin (\Phi)
$$

Since the roll and pitch angles are small during the whole flight, $D$ can be approximated as $D \approx \frac{h}{\cos (\varphi) \cdot \cos (\theta)}$, where $\varphi$ denotes the roll angle, $\theta$ denotes the pitch angle and $h$ denotes the local ground height. The rotational optic flow depends only on the angular speed $\Omega_{j}$ expressed in $\mathcal{B}$ associated with the vector basis $(\vec{x}, \vec{y}, \vec{z})$, where $j$ denotes the axis of rotation, and on the elevation angle $\lambda$ between the gaze direction and the axis of rotation which is always $\frac{\pi}{2}$ in the 2D case (see [24] for a graphical illustration).

$$
\omega_{R}=\Omega_{j} \sin (\lambda)
$$

In our case, $\Phi=\theta+\gamma+\frac{\pi}{2}$ (with the sensor oriented downward, $\gamma<0, \theta>0$ ), $\lambda=\frac{\pi}{2}$ and $\Omega_{j}=\Omega_{2}$, where $\Omega_{2}$ is the pitch angular velocity defined in $\mathcal{B}$, the approximate ground-truth optic flow is therefore computed as follows:

$$
\omega_{g r d-t r h}=\left(\frac{v}{h} \cdot \cos (\theta) \cdot \cos (\varphi) \cdot \sin \left(\theta+\gamma+\frac{\pi}{2}\right)\right)+\Omega_{2}
$$

where $\gamma$ denotes the angle between the orientation of the velocity vector $\vec{v}$ and the local horizontal plane. We computed the ground-truth optic flow as precisely as possible. But, since the data are coming from cartographic data previously recorded and from different sensors with different accuracy as well as different noise sources, $\omega_{\text {grd-trh }}$ is the approximate ground-truth optic flow. Fig. 1 shows the Inertial $\mathcal{I}$ and Body-fixed $\mathcal{B}$ frames and the main variables defining the optic flow. During the experiments described below, the approximate ground-truth optic flow $\omega_{g r d-t r h}$ was computed using data from the inertial measurement unit (IMU), the global positioning system (GPS) and the data grabbed by a LIDAR (Light Detection And Ranging) during previous flights over the same fields.

\section{iII. Presentation of the Low-Speed Visual Motion SENSOR}

The new low-speed visual motion sensor consists mainly of a low-cost plastic lens placed in front of an off-theshelf photosensor array. The photosensor used in this study, which is called the LSC, was purchased from iC-Haus: it features six photodiodes, each having a large sensitive area of $300 \times 1600 \mu \mathrm{m}$ and an integrated preamplifier. The LSC conveys the visual signals received to a hybrid analog/digital processing algorithm, where the optic flow value $\omega_{\text {meas }}$ is computed. The cheap, lightweight lens used here was a CAX183 from Thorlabs (focal length $18.33 \mathrm{~mm}$, f-number 4.07). A custom-made protective case was added in order to protect the low-weight sensor and the optical assembly from unfavorable weather conditions (see Fig. 2.a for pictures and Fig. 2.b for an exploded view). The new visual motion sensor and its custom-made protective case weighed 29.4g. Many of the parameters of the original visual motion detecting scheme presented in [13], [14] have been updated, especially in terms of the optical angles and the cut-off frequency of the temporal filters. The six optical axes formed by the photodiodes are separated by an interreceptor angle $\Delta \varphi$. By defocusing the lens (i.e., by adjusting the distance between the lens and the photosensors), we obtained Gaussian angular sensitivity functions for each photoreceptor with a correlation coefficient greater than $99 \%\left(R_{L S C}^{2}>0.990\right.$ see Fig. 3), in line with what occurs in the common fly's eye [25]. These features were assessed by slowly rotating the lens in front of a point light source placed at a distance of $85 \mathrm{~cm}$. The local 1$\mathrm{D}$ angular speed $\omega_{\text {meas }}$ measured by the sensor was defined as the ratio between the interreceptor angle $\Delta \varphi$ and the time elapsing $\Delta t$ between the moments when two adjacent photodiode signals reach the threshold (i.e., the time of travel of a contrast from the optical axis of one photodiode to the optical axis of the following one).

$$
\omega_{\text {meas }}=\frac{\Delta \varphi}{\Delta t}
$$




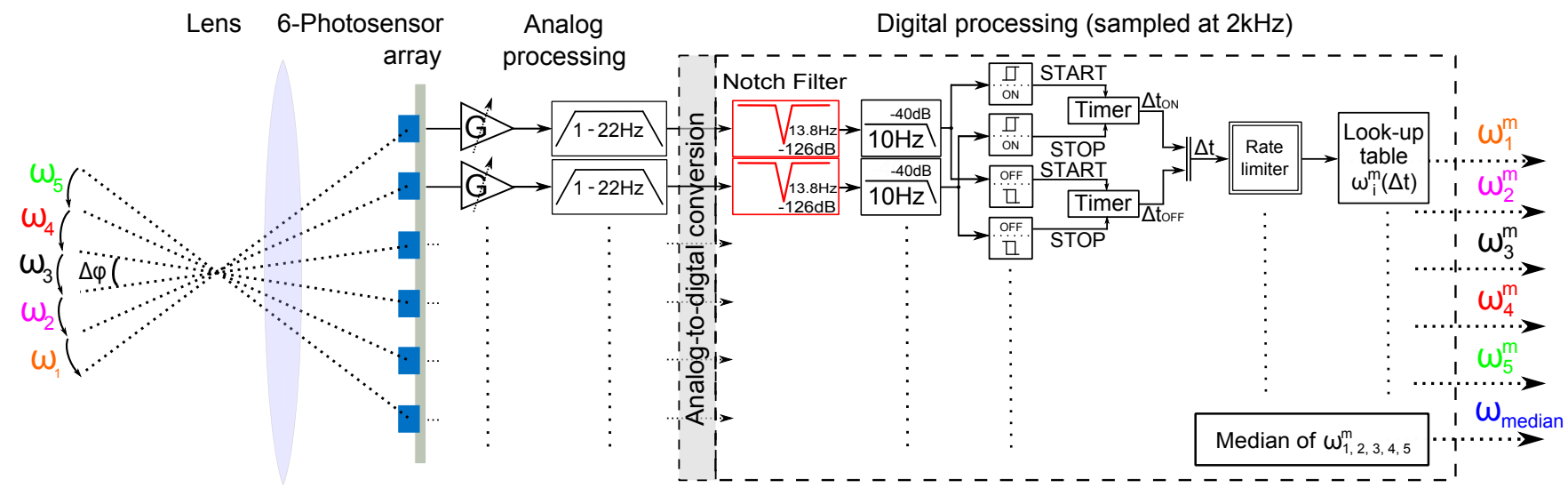

Fig. 4. General processing architecture of the low-speed visual motion sensor. First of all, the defocused lens carries out the spatial sampling and spatial low-pass filtering steps. The six photodiode signals are amplified by a programmable gain in order to increase the signal to noise ratio, before being filtered by an analog bandpass filter $(1-22 \mathrm{~Hz})$. The digital stage begins with a second order fixed-point notch filter centered on the main rotor frequency of ReSSAC, $13.8 \mathrm{~Hz}$. It is followed by a second order fixed-point low pass filter with a cut-off frequency set at $10 \mathrm{~Hz}$. A hysteresis thresholding process is associated with the computation of the time $\Delta t$ elapsing between two adjacent signals (with either ON or OFF contrasts). Lastly, after an outlier filtering step, the output signal of the 1-D visual motion sensor is obtained from a precomputed look-up table and the median value is calculated.

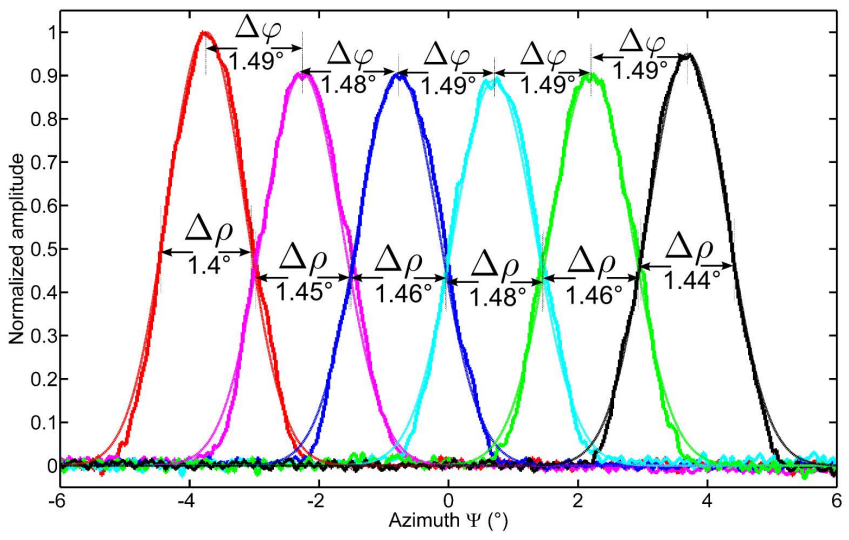

Fig. 3. Gaussian angular sensitivity functions of the LSC photosensor array with a CAX183 plastic aspherical lens, raw data (thick curves) and approximate Gaussian-shaped fit (thin curves). By adjusting the distance between the lens and the LSC photosensor, we obtained a correlation coefficient almost equal to $1\left(R_{L S C}^{2}>0.990\right)$, and a $\Delta \varphi$ value approximately equal to $\Delta \rho$.

In [11], the measurement range of the sensor covered a large range of high speeds from $50^{\circ} / \mathrm{s}$ to $300^{\circ} / \mathrm{s}$, whereas the present study focused on low velocities giving a range of $1.5^{\circ} / \mathrm{s}$ to $25^{\circ} / \mathrm{s}$, which is more than tenfold slower. In order to stay in the same range of $\Delta t$, whose accuracy of measurement depends on the microcontroller's sampling frequency, we therefore had to narrow $\Delta \varphi . \Delta \varphi$ corresponds to the angle separating two adjacent photodiodes optical axis: it depends on the focal lens, on the pitch (distance between the center of two adjacent photodiodes) and also on the distance from the photodiode plane to the focal point which is the easiest setting to adjust.

The large $18.33 \mathrm{~mm}$ focal length increases the defocalizing effects of the lens, giving a suitably small mean interreceptor angle of $\overline{\Delta \varphi}=1.488^{\circ}$. The second advantage of the defocusing process is that it adds a blurring effect giving
Gaussian-shaped angular sensitivity functions and change $\Delta \rho$. As found to occur in some diurnal insects [26],

$$
\Delta \varphi=\Delta \rho
$$

Achieving a tight $\Delta \rho$ made it possible for the sensor to respond to higher spatial frequency contrasts. The acceptance angle, defined by $\Delta \rho$, acts like an optical low pass spatial filter.

We eventually reached $1.4 \geq \Delta \varphi \approx \Delta \rho<1.5^{\circ}$, corresponding to a field of view in the direction of the visual motion sensor of $10.28^{\circ}\left(\sum_{i=1}^{5} \Delta \varphi_{i}+\frac{\Delta \varphi_{1}}{2}+\frac{\Delta \varphi_{5}}{2}\right)$. Table I gives the optical characteristics of the sensor. The general processing

\begin{tabular}{|c|c|}
\hline Focal length of the lens CAX183 [mm] & 18.33 \\
\hline$f_{\text {number }}$ of the lens [\#] & 4.07 \\
\hline Angular velocity range $[\%$ s] & {$[1.5 ; 25]$} \\
\hline Field of view of a single photodiode $\left[{ }^{\circ}\right]$ & $2.90 \times 15.44$ \\
\hline Sensitivity $[\% / \mathrm{s} / \mathrm{LSB}]$ & $4.58 \mathrm{e}-5$ \\
\hline Mean interreceptor angle $\overline{\Delta \varphi}\left[{ }^{\circ}\right]$ & 1.488 \\
\hline Mean acceptance angle $\overline{\Delta \rho}\left[{ }^{\circ}\right]$ & 1.448 \\
\hline Photodiode size $[\mu \mathrm{m}]$ & $300 \times 1,600$ \\
\hline Pixel pitch $[\mu m]$ & 420 \\
\hline Resolution [\%] [min; max] & {$[0.01 ; 0.21]$} \\
\hline $\begin{array}{l}\text { Mass of the visual motion sensor in a } \\
\text { stand-alone version }[\mathrm{g}]\end{array}$ & 2.8 \\
\hline
\end{tabular}

TABLE I

Characteristics OF THE NEW LOW-SPEED VISUAL MOTION SENSOR

algorithm consists of two parts: an analog processing part converts the six visual signals into electrical signals with a high signal to noise ratio, and the digital processing part then simultaneously computes five optic flow values plus the median value (see Fig. 4). The analog processing begins with a programmable gain connected to the microcontroller via a SPI communication bus [12]. A pass-band filter then differentiates the visual signal and acts as an anti-aliasing filter.

The digital processing algorithm starts with a second order 
fixed-point notch filter centered on the ReSSAC's main rotor frequency. The center frequency of the filter is $f_{0}=13.8 \mathrm{~Hz}$ with a Q-factor $Q=6.9$ at a sampling frequency $f_{s}=$ $500 \mathrm{~Hz}$. Its transfer function, which has been defined in [27], is as follows:

$$
H_{n o t c h}(z)=b \frac{1-2 \cos \left(\omega_{0}\right) z^{-1}+z^{-2}}{1-2 b \cos \left(\omega_{0}\right) z^{-1}+(2 b-1) z^{-2}}
$$

with

$$
b=\frac{1}{1+\frac{\sqrt{1-G_{B}^{2}}}{G_{B}} \tan \left(\frac{\Delta \omega}{2}\right)}
$$

where $\Delta \omega$ is the full width at a level $G_{B}^{2}$ and $\omega_{0}$ is the center frequency. We chose $\omega_{0}=2 \cdot \pi \frac{f_{s}}{f_{0}}, \Delta \omega=2 \cdot \pi \frac{\Delta f}{f_{s}}$ with $\Delta f=2 H z$ and $G_{B}^{2}=-3 d B$. As the visual angular speed $\omega_{\text {meas }}$ is quite low, the temporal frequency $f_{t}$ of the visual signal (which consists of contrasts) is also quite low, as expressed by the following equation [28]:

$$
f_{t}=\omega_{\text {meas }} \cdot f_{\text {spatial }}
$$

where $f_{\text {spatial }}$ is the spatial frequency (in cycles $/^{\circ}$ ) associated with the contrasting pattern. Therefore, a second order fixed-point low pass filter was used to enhance the signal to noise ratio by removing the noise remaining at frequencies of more than $10 \mathrm{~Hz}$.

The algorithm called the "Time of travel scheme" implemented here consists mainly of a hysteresis thresholding process with separate ON and OFF pathways [13], [14], [29]-[31] followed by the $\Delta t$ computation, the result of which is fed into a corresponding table. Lastly, the five simultaneously computed optic flows $\omega_{i}^{m}$ are combined by the median operator in order to increase the robustness and the refresh rate of the output [31].

The microcontroller used for this purpose is a dsPIC33FJ128GP802 working at a sampling frequency of $2 \mathrm{kHz}$, except for the digital filters, which are sampled at a rate of $500 \mathrm{~Hz}$. Special efforts were made to optimize the algorithm, and a computational load of only $17 \%$ was eventually obtained.

\section{ExPERIMENTAL RESUlts}

\section{A. Dynamic visual motion characteristics on the ground}

The characteristics of the present visual motion sensor (VMS) were assessed by performing optic flow measurements under controlled motion conditions (orientation and velocity) outdoors on the ground. Pure rotational motion was applied to the sensor with angular speed variations ranging from $1^{\circ} / \mathrm{s}$ to $20^{\circ} / \mathrm{s}$ using a previously described outdoor ground-based set-up [11]. The triangular response pattern obtained corresponds closely to the reference angular speed (see Fig. 5). It can therefore be said that this tiny 6-pixels sensor is able to accurately compute the 1-D visual angular speed within its operating range. The refresh rate is defined as the ratio between the total number of new measurements of each $\omega_{i}$ occurring within the acceptable range $\left[1.5^{\circ} / \mathrm{s}\right.$ $\left.25^{\circ} / \mathrm{s}\right]$ and the time elapsing. The median value is delivered at $2 \mathrm{kHz}$ (output data rate) even if the measure is not

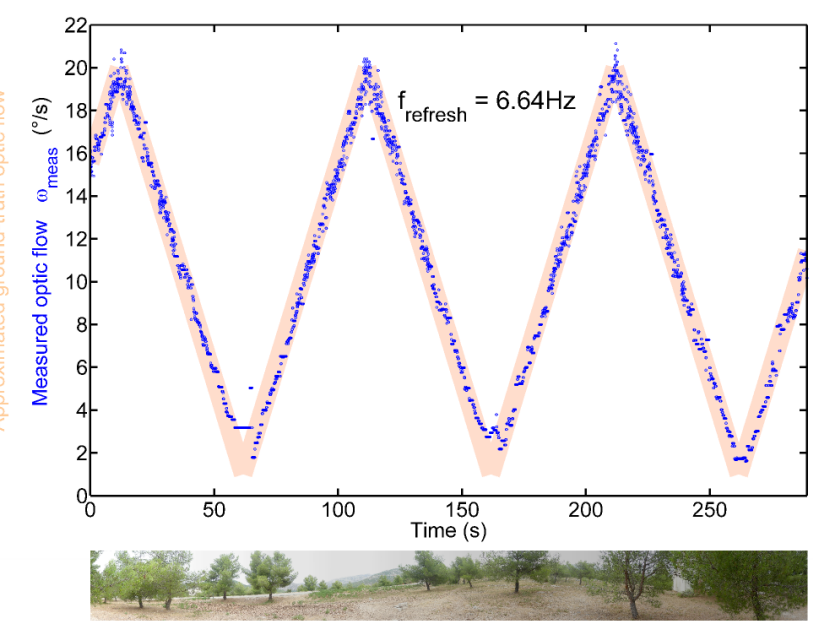

Fig. 5. Dynamic outdoor response of the low-speed VMS (blue), as compared with the ground-truth optic flow (red). The visual motion sensor was rotated by means of a conveyor belt driven by a stepping motor (103H5208-0440 from Sanyo-Denki) [11]. Rotations from $1^{\circ} / \mathrm{s}$ to $20^{\circ} / \mathrm{s}$ were applied to the sensor, which is designed to operate in the $1.5^{\circ} / \mathrm{s}$ to $25^{\circ} / \mathrm{s}$ range. The optic flow measured closely matched the reference signal, with a refresh rate of $6.64 \mathrm{~Hz}$. Since no synchronization signal was available, the ground-truth optic flow has been roughly synchronized here.

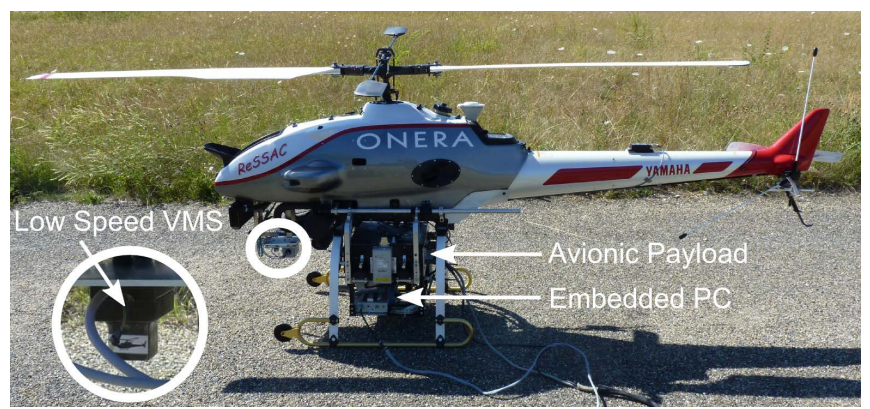

Fig. 6. The ONERA ReSSAC Unmanned Autonomous Helicopter before takeoff, featuring the on-board low-speed VMS mounted at the front end looking downward

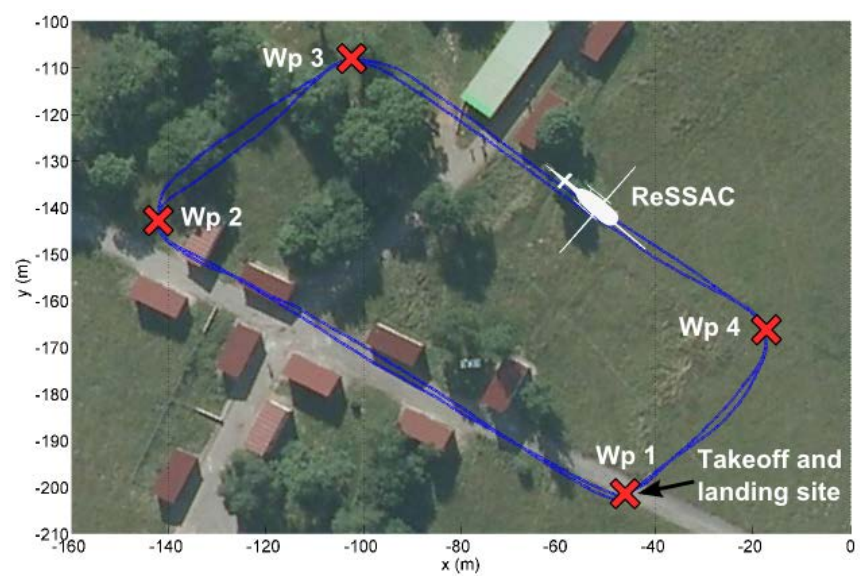

Fig. 7. Top view of the trajectory taken by ReSSAC, as defined by 4 GPS waypoints. The UAV flew over a complex textured environment containing houses, trees, roads and a sloping field. The UAV was controlled manually during the takeoff and landing phases (these parts of the trajectory are not shown on the figure). Aerial view obtained on geoportail.fr. 

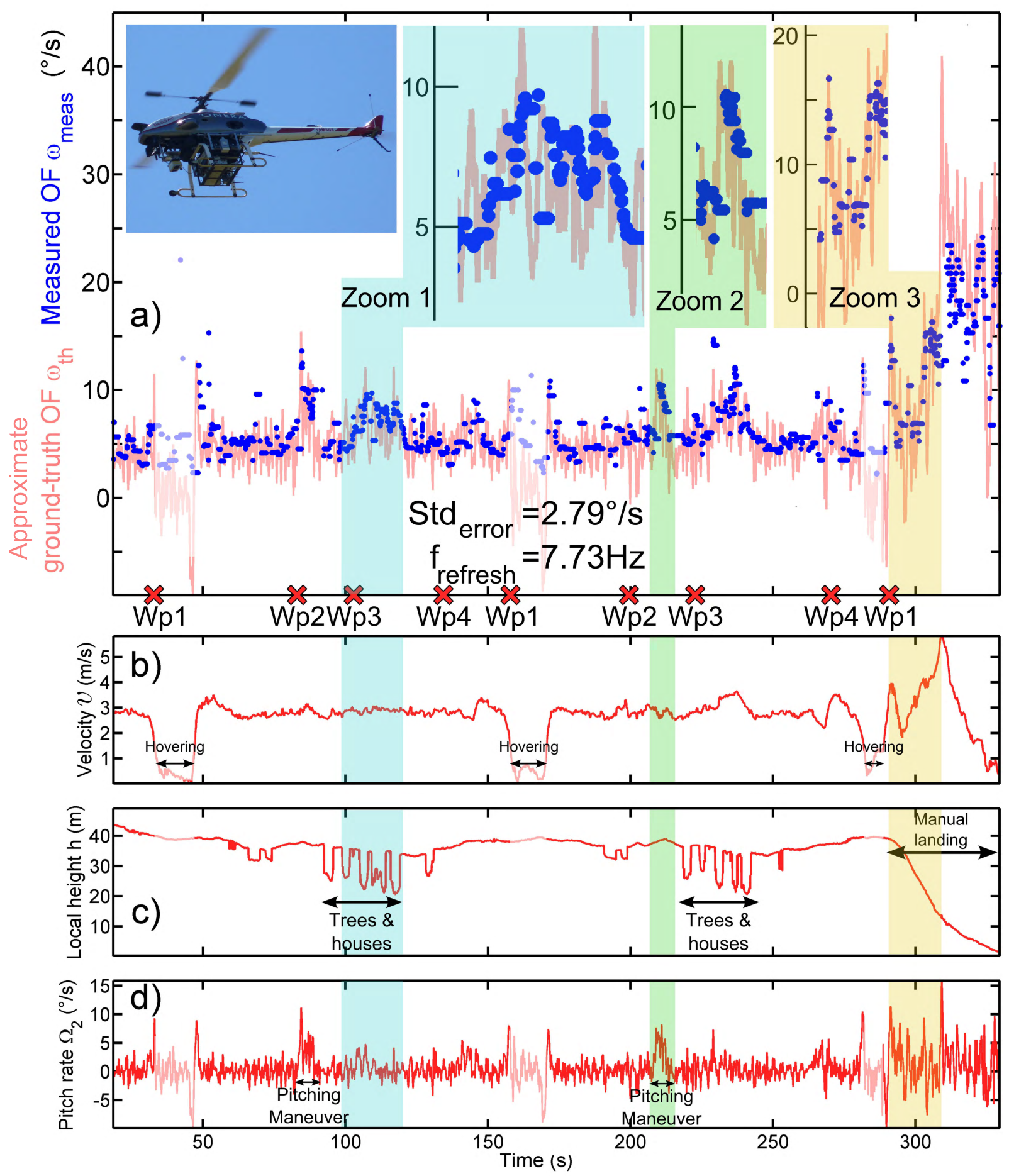

Fig. 8. Low-speed visual motion sensor and flight data sensed on-board the ReSSAC UAV. (a) Ground-truth optic flow (see (4)) (red) and measured optic flow $\omega_{\text {meas }}=\Delta \varphi / \Delta t$ (blue dots). Despite the strong variations mainly due to vibrations, the low-speed visual motion sensor's output closely matched the ground-truth optic flow, giving a standard deviation of $2.79^{\circ} / \mathrm{s}$ and a refresh rate of $7.73 \mathrm{~Hz}$. The first area highlighted (Zoom 1) shows the effects of strong variations in the local height due to the successive trees and houses: the low-speed VMS sensed these height variations. After reaching Wp2 in the second lap (inside the second area highlighted), one can see a strong pitch angular speed peak, which is again directly reflected in the low-speed VMS measurement signal (Zoom 2). Lastly, during the manual landing phase (Zoom 3), as the forward speed increased and the local height decreased, both the ground-truth and sensed optic flows increased sharply and the measurements were still accurate (the standard deviation was not computed during the landing phase or while hovering because the ground-truth optic flow was not entirely in the sensor's measurement range). (b) Norm of the velocity vector during the trajectory. (c) Local ground height measured by combining GPS data (OEM4 G2 from NovAtel) and previously mapped LIDAR data (Sick LDMRS 400001). The nominal height was around $40 \mathrm{~m}$. But due to the variable relief, the local height often changed suddenly by 15 meters. (d) Pitch rate of ReSSAC as measured by the IMU. 

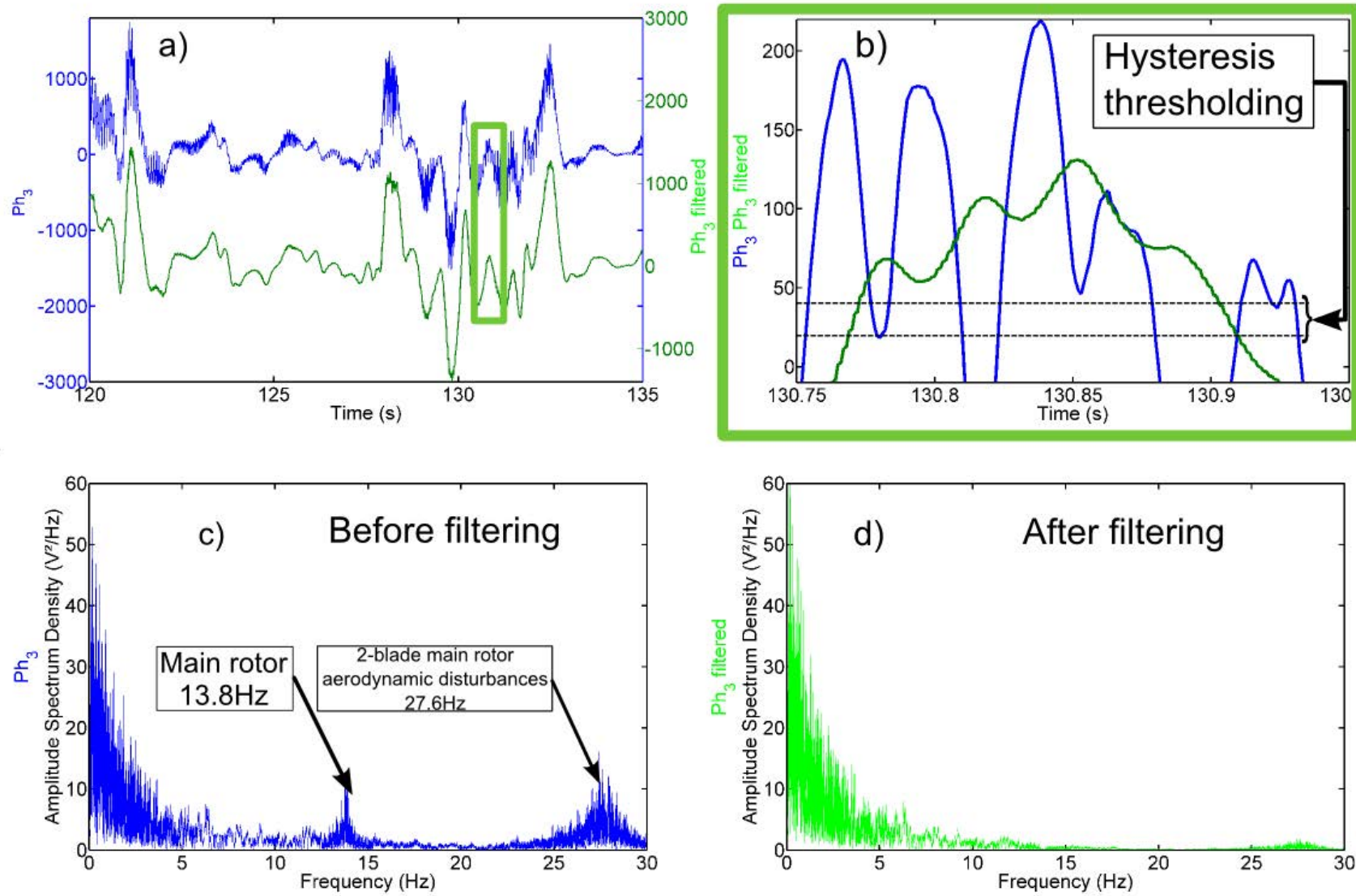

Fig. 9. Visual signal before and after the digital fixed point filtering step (notch and low pass filter): (a) Large view of the signals before (blue) and after (green) the filtering. (b) Zoom on the signals. Without any filtering, the visual motion sensor would have detected contrasts whenever, the signal was greater than 40 and then decreased to less than 20 (because of the hysteresis thresholding). One can see that the filtered signal detected only one contrast during this phase. (c) Power spectrum of the 3rd photosensor sampled at $1 \mathrm{kHz}$ before (blue) and after the filtering (green). The rotor's main rotational speed was $828 \mathrm{rpm}$ (i.e. $13.8 \mathrm{~Hz}$ ): this disturbance was filtered out by adding a second-order notch filter.

refreshed, that is why the refresh rate metric is needed to evaluate the performance of the sensor. The mean refresh rate achieved during the dynamic performances evaluation was $f_{\text {refresh }}=6.64 \mathrm{~Hz}$ : this value depends on the richness of the visual environment, as well as on the actual angular speed.

\section{B. Free-flying helicopter's trajectories}

The low-speed VMS performances were studied on a freeflying UAV during forward flight over fields. The ONERA's ReSSAC unmanned helicopter was used to test the sensor's dynamic responses. The characteristics of ReSSAC (Yamaha $\mathrm{RmaX}$ ) in terms of the mass balance have been described in [32]. Its mass, its flight envelope and the vibration dynamics due to the main rotor's rotational speed presented us with quite a challenging ground-truth optic flow profile. The lowspeed visual motion sensor was embedded at the front end of ReSSAC pointing directly downward with a clear field of view (see Fig. 6). The sensor was connected to the embedded PC (pip22 from MPL) via a serial port. VMS, IMU and GPS data were logged locally onto the embedded PC with a system clock timestamp for subsequent processing purposes. A WiFi network was used to set and adapt the programmable gain and the threshold value during the experiments, via a graphical user interface displaying raw visual signals and optic flow signals on the ground station. The trajectory to be taken was set offline in the form of a parallelogramlike shape defined by four GPS waypoints called Wp1 to Wp4 (see Fig. 7). The experiment was carried out over an uninhabited village providing a very rich and complex visual environment consisting of various components:

- From $\mathrm{Wp} 1$ to $\mathrm{Wp} 2$ : roads and buildings,

- From Wp2 to Wp3: trees,

- From Wp3 to Wp4: trees, road, a sloping green field,

- From Wp4 to Wp1: road, a green field.

Data logging started at the beginning of the scenario and ended after landing. During the automatic phase, ReSSAC's flight envelope was tightened to ensure the helicopter's safety, whereas in the manual mode, the human pilot was able to reach greater speeds at lower heights. According to (4), increasing the velocity while decreasing the altitude will increase the ground-truth optic flow $\omega_{\text {grd-trh }}$. The flight was performed in South-western France in mid-July around $5 \mathrm{pm}$ on a bright sunny day: the mean illuminance was approximately $10000 l x$. 


\section{Free-flying results}

Fig. 8 shows an accurate response of the low-speed visual motion sensor mounted onboard the unmanned ReSSAC helicopter. Despite the complex ground-truth optic flow, the visual motion sensor responded appropriately to the visual stimuli. The standard deviation of the error between the approximate ground-truth optic flow $\omega_{\text {grd-trh }}$ and the measured optic flow $\omega_{\text {meas }}$ was less than $2.8^{\circ} / \mathrm{s}$, which is quite low. The standard deviation was computed only during automatic flight phases. Measurements performed during hovering flight phases (i.e. $\omega_{g r d-t r h}$ outside the measurement range) were rejected offline (white blurred zones). The refresh rate $f_{\text {refresh }}$ was greater than $7.7 \mathrm{~Hz}$, which is even slightly higher than in the dynamic measurements performed during a rotating motion on ground-based set-up (see Fig. 5). Fig. 8.b, 8.c, 8.d giving the velocity norm, the local ground height and the pitch angular speed, show how well the sensor responded to its visual environment. Three areas of particular interest (highlighted areas in Fig. 8) emerge from these results:

Zoom 1: ReSSAC helicopter flying over houses and trees, giving several sudden changes in the local height (nominal height during flight: $35-40 \mathrm{~m}$ ). Once again, the low-speed VMS accurately sensed these height variations and yielded similar values to the approximate ground-truth value.

Zoom 2: ReSSAC helicopter pitched forward after Wp2 (see Fig. 8.d), which produced a series of high angular speed variations around $5^{\circ} / \mathrm{s}$. These variations in the visual motions are directly reflected in the low-speed VMS measurement output signal, which closely matched the approximate groundtruth optic flow pattern. This rotational optic flow occurs on the right hand side of (4) and corresponds to $\Omega_{2}$.

Zoom 3: ReSSAC helicopter was piloted manually during the landing phase, which significantly increased the speed and decreased the height. Both the approximate ground-truth and sensed optic flows reflect this sharp increase. The results obtained during this phase are interesting because they show the performances of the visual sensor over its whole measurement range.

The robust and accurate performances observed during this experiment show that the low-speed visual motion sensor is highly suitable for use in many high-scaled robotic applications. A close look at the raw (blue) and filtered (green) photodiode signals (see Fig. 9.a-d) shows the value of the filtering stage, which reduces the noise induced by the helicopter's main rotor. One can see the high spectrum density occurring around $13.8 \mathrm{~Hz}$ and $27.6 \mathrm{~Hz}$ on the raw signal (blue) and the low spectrum density on the filtered one (green) (see Fig. 9.c and 9.d). The whole body of the rotorcraft was vibrating continuously around its equilibrium point at the higher angular speeds, resulting in fairly strong oscillations in the unfiltered visual signals. The noise due to rotor-vortex interactions in the main 2-blade rotor (see [33] for further details about helicopters' aerodynamic disturbances) was even stronger that the main rotor's fundamental frequency, and it is therefore proposed to add another dedicated notch filter centered on $27.6 \mathrm{~Hz}$ in future studies.

\section{CONCLUSION}

A 6-pixel visual motion sensor dedicated to low visual angular speeds was developed and successfully tested outdoors onboard a free flying unmanned helicopter under real-life dynamic and vibratory conditions as a means of measuring local 1-D angular speeds ranging from $1.5^{\circ} / \mathrm{s}$ to $25^{\circ} / \mathrm{s}$. The results obtained here show that the 6-pixel visual motion sensor is able to accurately sense low visual angular speeds, giving quite frequently refreshed measurements even at high heights over an unknown complex outdoor environment. Among the large range of potential applications to which this 6-pixel sensor lends itself, landing on extra-planetary bodies immediately comes to mind. Soft lunar landing involves a similar optic flow range to that studied here [34]-[36], with demanding final conditions in terms of the longitudinal and vertical velocities, which makes this new visual motion sensor a good candidate for applications of this kind, given the promising results obtained here. In conclusion, this lightweight, low cost, low power and minimalist visual motion sensor also seems to be well suited for equipping UAV helicopter autopilots designed to perform hazardous tasks such as outdoor landing/takeoff and obstacle avoidance.

\section{ACKNOWLEDGMENTS}

We are most grateful to S. Viollet, L. Burlion, E. Kervendal, E. Bornschlegl, F. Roubieu and F. Expert for their fruitful suggestions and comments during this study. We thank H. de Plinval, A. Amiez and V. Fuertes for the successful flight of ReSSAC, T. Rakotomamonjy and A. Piquereau for their expertise in helicopter dynamics, $\mathbf{M}$. Boyron for his assistance with the electrical design, J. Diperi for his involvement in the mechanical design and J. Blanc for improving the English manuscript.

\section{REFERENCES}

[1] B. Hérissé, T. Hamel, R. Mahony, and F.-X. Russotto, "Landing a VTOL unmanned aerial vehicle on a moving platform using optical flow," IEEE Transactions on Robotics, vol. 28, no. 1, pp. 77 -89, February 2012.

[2] G. Barrows and C. Neely, "Mixed-mode VLSI optic flow sensors for in-flight control of a Micro Air Vehicle," in in SPIE : Critical technologies for the future of computing, vol. 4109, San Diego, CA, USA, Aug 2000, pp. 52-63.

[3] S. Griffiths, J. Saunders, A. Curtis, B. Barber, T. McLain, and R. Beard, "Maximizing miniature aerial vehicles," IEEE Robotics \& Automation Magazine, vol. 13, pp. 34-43, 2006.

[4] A. Beyeler, J. Zufferey, and D. Floreano, "OptiPilot: control of takeoff and landing using optic flow," in European Micro Aerial Vehicle Conference (EMAV), vol. 27, Delft, Nederlands, September 2009.

[5] M. Garratt and J. Chahl, "Vision-based terrain following for an unmanned rotorcraft," Journal of Field Robotics, vol. 25, pp. 284-301, 2008.

[6] F. Kendoul, K. Nonami, I. Fantoni, and R. Lozano, "An adaptive vision-based autopilot for mini flying machines guidance, navigation and control," Autonomous Robots, vol. 27, pp. 165-188, 2009. 
[7] N. Franceschini, A. Riehle, and A. Nestour, Facets of vision. Springer, 1989, ch. Directionally selective motion detection by insect neurons, pp. 360-390.

[8] N. Franceschini, J. Pichon, and C. Blanes, "From insect vision to robot vision," Philosophical Transactions of the Royal Society of London, vol. 337, pp. 283-294, 1992.

[9] F. Ruffier and N. Franceschini, "Visually guided micro-aerial vehicle: automatic take off, terrain following, landing and wind reaction," in IEEE International Conference on Robotics and Automation (ICRA), vol. 3, April 2004, pp. 2339 - 2346.

[10] N. Franceschini, F. Ruffier, and J. Serres, "A bio-inspired flying robot sheds light on insect piloting abilities," Current Biology, vol. 17, pp. 329-335, 2007.

[11] F. Expert, S. Viollet, and F. Ruffier, "Outdoor field performances of insect-based visual motion sensors," Journal of Field Robotics, vol. 28, pp. 529-541, 2011.

[12] F. Ruffier and F. Expert, "Visual motion sensing onboard a 50-g helicopter flying freely under complex VICON-lighting conditions," in International Conference on Complex Medical Engineering, Kobe, Japan, July 2012, pp. 634-639.

[13] C. Blanes, "Appareil visuel élémentaire pour la navigation à vue d'un robot miobile autonome," (Advisor: N. Franceschini), Neurosciences, Univ. Aix-Marseille II, 1986.

[14] J.-M. Pichon, C. Blanes, and N. Franceschini, "Visual guidance of a mobile robot equipped with a network of self-motion sensors," in SPIE Conf. on Mobile Robots IV, W. Wolfe and W. Chun, Eds., vol. 1195, Bellingham, U.S.A., 1989, pp. 44-53.

[15] R. Benson and T. Delbrück, Direction selective silicon retina that uses null inhibition, D. S. Touretzky, Ed. San Mateo, CA: Morgan Kaufman, 1992.

[16] R. Moeckel and S.-C. Liu, "Motion detection circuits for a time-totravel algorithm," in IEEE International Symposium on Circuits and Systems (ISCAS), may 2007, pp. 3079-3082.

[17] W. Green, P. Oh, and G. Barrows, "Flying insect inspired vision for autonomous aerial robot maneuvers in near-earth environments," in International Conference on Robotics and Automation (ICRA), vol. 3, april-1 may 2004, pp. 2347-2352 Vol.3.

[18] S. Hrabar, G. Sukhatme, P. Corke, K. Usher, and J. Roberts, "Combined optic-flow and stereo-based navigation of urban canyons for a uav," in International Conference on Intelligent Robots and Systems (IROS), aug. 2005, pp. 3309-3316.

[19] J. Conroy, G. Gremillion, B. Ranganathan, and J. Humbert, "Implementation of wide-field integration of optic flow forautonomous quadrotor navigation," Autonomous Robots, vol. 27, pp. 189-198, 2009.

[20] Y. Watanabe, P. Fabiani, and G. Le Besnerais, "Simultaneous visual target tracking and navigation in a gps-denied environment," in International Conference on Advanced Robotics (ICAR), june 2009, pp. 1 -6 .

[21] A. Beyeler, J. C. Zufferey, and D. Floreano, "Vision-based control of near-obstacle flight," Autonomous robots, vol. 27, pp. 201-219, 2009.

[22] F. Kendoul, I. Fantoni, and K. Nonamib, "Optic flow-based vision system for autonomous 3d localization and control of small aerial vehicles," Robotics and Autonomous Systems, vol. 57, pp. 591-602, 2009.

[23] J. Koenderink and A. Doorn, "Facts on optic flow," Biological Cybernetics, vol. 56, pp. 247-254, 1987.

[24] F. Ruffier, "Pilote automatique biomimétique. Système générique inspiré du contrôle visuomoteur des insectes pour: le suivi de terrain, la réaction au vent et l'atterrissage automatiques d'un micro-aéronef,' Ph.D. dissertation, Institut National Polytechnique de Grenoble, 2004.

[25] K. Götz, "Optomotorische untersuchung des visuellen systems einiger augenmutanten der fruchtfliege drosophila." Biological Cybernetics, vol. 2, pp. 77-92, 1964.

[26] M. F. Land, "Visual acuity in insects," Annual Review of Entomology, vol. 42, pp. 147-177, 1997.

[27] S. J. Orfanidis, Introduction to signal processing, P.-H. Inc, Ed. Prentice-Hall, Inc. Upper Saddle River, NJ, USA, 1995.

[28] A. Landolt and A. Mitros, "Visual sensor with resolution enhancement bymechanical vibrations." Autonomous Robots, vol. 11 (3), pp. 233239, 2001.

[29] F. Ruffier, S. Viollet, S. Amic, and N. Franceschini, "Bio-inspired optical flow circuits for the visual guidance of micro air vehicles." in IEEE International Symposium on Circuits and Systems (ISCAS), vol. 3, Bangkok, Thailand, 2003, pp. 846-849.
[30] F. Ruffier and N. Franceschini, "Optic flow regulation: the key to aircraft automatic guidance," Robotics and Autonomous Systems, vol. 50, pp. 177-194, 2005.

[31] F. Roubieu, F. Expert, M. Boyron, B. Fuschlock, S. Viollet, and F. Ruffier, "A novel 1-gram insect based device measuring visual motion along 5 optical directions," in IEEE Sensors conference, Limerick, Ireland, 2011, pp. 687-690.

[32] Y. Watanabe, C. Lesire, A. Piquereau, P. Fabiani, M. Sanfourche, and G. Le Besnerais, "The ONERA ReSSAC unmanned autonomous helicopter: Visual air-to-ground target tracking in an urban environment," in American Helicopter Society 66th Annual Forum, Phoenix, AZ, USA, 2010

[33] A. Bramwell, G. Done, and D. Balmford, Helicopter Dynamics, 2nd ed. London: AIAA, Butterworth-Heinemann, 1986.

[34] D. C. Cheatham, F. V. Bennett, and T. M. Branch, "Apollo lunar module landing strategy," in Proceedings of the Apollo Lunar Landing Mission Symposium, 1966.

[35] F. Valette, F. Ruffier, S. Viollet, and T. Seidl, "Biomimetic optic flow sensing applied to a lunar landing scenario," in International Conference on Robotics and Automation (ICRA), 2010, pp. 2253-2260.

[36] D. Izzo, N. Weiss, and T. Seidl, "Constant-optic-flow lunar landing: Optimality and guidance," Journal of Guidance, Control, and Dynamics, vol. 34, pp. 1383-1395, 2011. 